\title{
Use of a Novel Panel of Monoclonal Antibodies against CENP-F for the Analysis of Cancerous Cells
}

Karlin A. Compton ${ }^{1}$, Emily L. Mace ${ }^{1}$ (co first author), Elise R. Pfaltzgraff ${ }^{1}$, Scott C. Borinstein ${ }^{2}$ and David M. Bader ${ }^{1}$

1. Division of Cardiovascular Medicine, Department of Medicine, Vanderbilt University School of Medicine, Nashville, Tennessee

2. Division of Pediatric Oncology, Department of Pediatrics, Vanderbilt University School of Medicine, Nashville, Tennessee

Centromere Protein F (CENP-F) is a very large (380 kilodalton) protein with multiple cellular activities including, but not restricted to, mitosis, pocket protein cycling, and Golgi function (1-3). Loss of CENPF function leads to multiple developmental defects and, in the case of the human gene, prenatal death $(4,5)$. Additionally and important to the current study, over-expression of CENP-F is thought to be a leading indicator of poor prognosis of specific cancers (6). With this in mind, we have begun an in depth analysis of CENP-F protein expression in cancerous cells. Specifically, we generated a novel panel of 10 independent monoclonal antibody-secreting cell lines directed against CENP-F to explore variations in expression levels in various cancer settings.

As a preliminary step in this effort, we examined CENP-F expression in HCT116 colorectal carcinoma and SW480 colorectal adenocarcinoma cells using quantitative immunofluorescence microscopy. Importantly, our data show that CENP-F expression is, in general, elevated in these cancerous cells when compared to expression levels of CENP-F in non-cancerous cells. Still greater complexity in these outcomes was revealed with the implementation of this novel, multi-antibody approach. First, immunofluorescent intensities generated by members of the monoclonal antibody panel varied significantly when reacted with HCT116 or SW2480 cells with specific reagents reacting at high levels while others were much less intense. Second, variation in the cellular distribution of immunofluorescent signal was also observed in the panel within each cell line. Thus, while elevation of CENP-F production in these two cancer cell lines was observed, use of multiple independent immunochemical reagents revealed a greater complexity in protein expression and distribution.

The proximate impact of the current studies is that this new panel of monoclonal antibodies may serve as a valuable reagent in predicting prognosis outcomes in various cancers. In a larger sense, the quantitative variation in CENP-F staining seen with these reagents demonstrates that multiple and sustainable immunochemical reagents must be generated for the analysis of protein expression in both basic and clinical settings. 


\section{References:}

[1] Feng, J et al. Chromosoma (2006)115:320-9.

[2] Ashe, M et al. J. Biol. Chem. (2004)279:664-76

[3] Moynihan, KL et al. Mol. Biol. Cell (2009) 20:4790-803.

[4] Dees, E et al. Cardiac-specific deletion of the microtubule-binding protein CENP-F causes dilated cardiomyopathy. (2012)5:468-80.

[5] Waters, AM et al. J. Med. Genet. (2015) 52:147-56.

[6] Cao, JY et al. Mol. Cancer (2010)9:237-50.
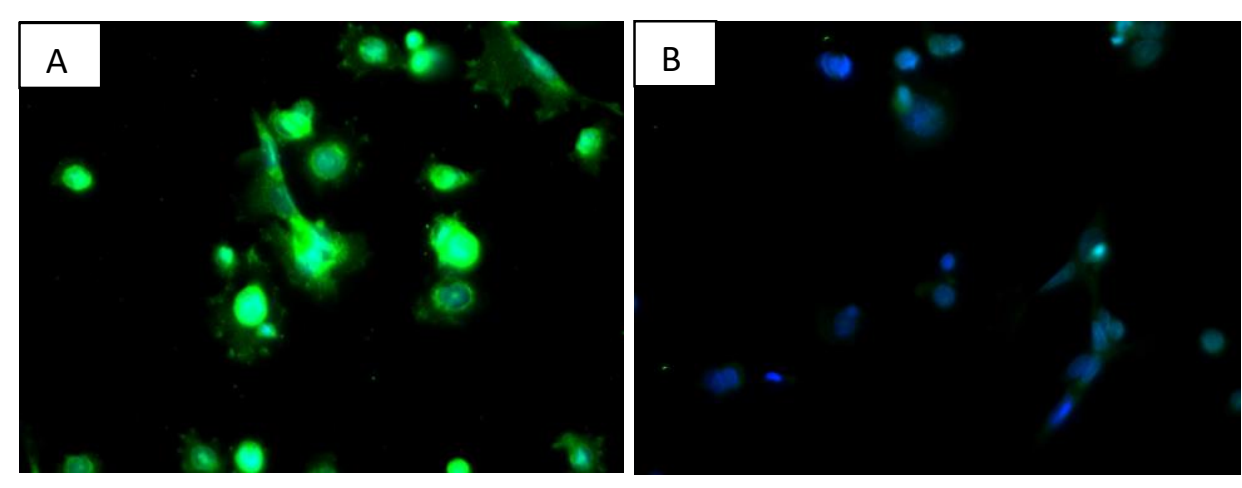

Figure 1. Reaction of two anti-CENP-F monoclonal antibodies demonstrates the variability of reaction in the same cell line. Here, SW480 cells were reacted and processed identically with two antibodies of our 10 antibody panel. While both antibodies bind the same CENP-F molecule, albeit in different domains, the florescent intensities are obviously very different. Interestingly, these antibodies bind HCT116 at nearly the same levels. 